\title{
Le paysage entre la Nature et la Société
}

\section{Georges Bertrand}

\section{Citer ce document / Cite this document :}

Bertrand Georges. Le paysage entre la Nature et la Société. In: Revue géographique des Pyrénées et du Sud-Ouest, tome 49, fascicule 2, 1978. Géosystème et aménagement. pp. 239-258;

doi : https://doi.org/10.3406/rgpso.1978.3552

https://www.persee.fr/doc/rgpso_0035-3221_1978_num_49_2_3552

Fichier pdf généré le 27/10/2018 


\begin{abstract}
Landscape between nature and society. Though it plays an essential part in our daily life, the landscape does not belong to any scientific category and it cannot be reduced to any single concept. The author proposes to study the landscape as a conception that is related to both nature and society sciences. Then landscape is defined as a social interpretation of a real fact which does exist without any consideration of the observer's will ; such a social interpretation cannot be separated from the economic and cultural production system to which the observer belongs. Thence any landscape should be examined at the same time as a subject and as an object as well, or, more accurately, as a permanent interchange between these two categories. Only such dynamic complex analysis of the landscaping process enables the scientist to draw the landscapes' sketches that can be used to define the landscape as a socio-ecological system and to recognize its function on the social process as a whole.
\end{abstract}

\title{
Résumé
}

Le paysage, qui occupe une place essentielle dans la vie quotidienne, ne relève d'aucune catégorie scientifique et n'est réductible à aucun concept. On a choisi d'étudier le paysage à la charnière des sciences de la nature et des sciences de la société. Il est défini comme une interprétation sociale, à partir d'un système de production économique et culturel, d'un objet matériel qui existe indépendamment de l'observateur. Le paysage doit donc être appréhendé en même temps comme sujet et comme objet, ou plus exactement comme le passage incessant de l'un à l'autre. Cette analyse complexe et dynamique du " processus " paysager permet d'esquisser les scénarios paysagers qui, d'une part, permettent de mieux cerner le paysage comme "système " socio-écologique et, d'autre part, de mieux l'insérer dans une démarche sociale. 
REVUE GÉOGRAPHIQUE DES PYRÉNÉES ET DU SUD-OUEST TOME 49 , FASC. 2 , pp. 239-258, Toulouse, 1978

\title{
Le paysage \\ entre la Nature et la Société \\ par Georges Bertrand *
}

\begin{abstract}
Pourquoi débattre encore du banal paysage ? S'agit-il, par souci disciplinaire, de préserver l'objet désuet qui est aussi le décor d'une géographie confinée ? S'agit-il de sacrifier à la vague écologique qui envahit l'analyse sociale ? Ou de répondre à une demande encore confuse et ambiguë et à de nouvelles questions sur les rapports que la société entretient avec l'espace ? S'agit-il de réfléchir à l'un des aspects des relations entre nature et culture au sein de la combinaison sociale ? Ou bien de s'attaquer à une notion idéologiquement opaque, récupérée et abâtardie par le discours dominant des mass media et par la pratique technocratique? Le problème du paysage est actuellement dans toutes ces questions et bien d'autres encore. Toutes ces interrogations et tous ces éléments disparates sont en fait indissociables mais, pour l'instant, inextricables.

La question du paysage paraît souvent le type même du débat impossible, voire du faux débat. Inanité scientifique, mais pourtant existence matérielle et culturelle quotidienne et inéluctable. Telle contradiction justifie à elle seule cette esquisse méthodologique. Pourquoi le paysage a-t-il été évacué aussi bien de la problématique sociale que de la problématique naturaliste ? Certes, le paysage n'est pas affaire de spécialiste et encore moins de discipline scientifique constituée. Il n'est pas de "science du paysage", au sens
\end{abstract}

\footnotetext{
* Professeur de Géographie à l'Université de Toulouse-Le Mirail.
} 
large du terme. Il n'y en aura sans doute jamais. Faut-il envisager une démarche spécifique, mais avec quelle finalité, à partir de quelle axiomatique et avec quelle méthodologie?

\section{L'idéologie du paysage : l'émergence et le refus}

Partir d'une définition analytique et rigoureuse du paysage serait supposer le problème résolu et il le serait alors de façon unilatérale. Paysage, un mot vivant, quotidien, que chacun utilise à sa guise et qui peut sembler vieillot, insipide, ou bien trop romantique. Il faut se laisser guider par l'usage social, et son brusque renouvellement qui en fait un mot fort et mobilisateur, un maître-mot de la société actuelle. Mass media, discours politico-technocratique, vocabulaire scientifique ensuite, multiplient les références à un paysage protéiforme, indéterminé, indéterminable. Paysage, paysager, étiquettes de garantie, accolés à n'importe quel produit à vendre : résidence secondaire, quartier neuf, bureau d'affaires, espace vert... ou publication scientifique. A la limite, le mot ne participe plus que du flou publicitaire. Il serait facile de relever le snobisme et la malhonnêteté d'une telle pratique, chez les uns et les autres, promoteurs ou chercheurs... Une idéologie du paysage s'est-elle constituée ? Ou bien, le paysage participe-t-il du confusionnisme de l'idéologie dominante ? Mais, l'émergence sociale du paysage se rattache à la vague écologico-environnementaliste qui secoue la société de consommation. Riche de telles contradictions et de son irréductible globalité, le paysage est devenu un enjeu politique : son analyse scientifique se pose autant en termes de pouvoir que de savoir.

On s'explique maintenant mieux le refus assez général de considérer le paysage comme un objet de recherche. On lui oppose des arguments scientifiques. Par sa complexité, par sa transgression du champ naturel au champ social, il s'exclut de lui-même des catégories scientifiques traditionnelles: ce n'est pas un concept et il ne peut le devenir. Aucune méthode connue ne saurait s'appliquer à une notion aussi confuse et chaotique, car le paysage n'est qu'un agglomérat d'objets disparates et très complexes qui ont individuellement sur lui l'avantage d'une existence scientifique fondée sur des disciplines connues, de la géologie à l'architecture. Surtout, le paysage se limite au visible, donc au perçu : il n'est que l'apparence des choses, les mécanismes étant, eux, invisibles. Ajoutons-y des arguments politiques : le paysage devient alors un produit de récupération ou d'agitation, une opération idéologique sans fondement scientifique. Il est significatif, à ce propos, de voir converger des opinions habituellement étrangères sinon opposées. Le paysage est alors l'objet d'exécutions sommaires : résurgence de la géographie descriptive classique, vision pseudo-scientifique d'écologistes 
délirants, machination technocratique destinée à masquer les « vrais » problèmes de la société. Manipulations douteuses, en particulier de ceux qui ont toujours craint d'être en retard d'une idée, d'un concept... ou d'un paysage. Le paysage est ainsi écrasé entre trop de sagesse et trop de folie, ou, plus prosaïquement, entre le mépris et l'imposture.

\section{Inadéquation des méthodologies naturalistes}

\section{Disqualification de la géographie classique.}

Rien de plus familier au géographe que le paysage concret et sa description heureusement balancée. Rien de plus étranger à la géographie que l'analyse globale et méthodique du paysage. Cette disqualification de la géographie classique correspond à deux lacunes fondamentales du projet géographique: d'une part, absence de réflexion théorique sur le concept de nature et de paysage (1); d'autre part, absence d'organisation de la monographie régionale.

Au point de départ, on trouve la géographie physique triomphante des années 1950-1960, dominée par une géomorphologie en plein essor méthodologique et de plus en plus autonome. Car le développement tardif et marginal de la Climatologie et de la Biogéographie modifie peu la hiérarchisation sectorielle du savoir géographique écrasé par la problématique de la Géomorphologie. L'étude géomorphologique masque le paysage ou le dévitalise.

Em. de Martonne, et surtout ses émules, ont mis à l'écart le projet humaniste et historisant que P. Vidal de la Blache fondait sur l'étude des paysages géographiques dans leur utilisation humaine. A partir de là les études sectorielles se développent en parallèle, approfondissent chacune leur domaine, n'apportant aucune contribution volontaire et réfléchie à la consolidation de ce qui n'est déjà plus une "synthèse géographique ». Les tentatives de Max. Sorre qui propose une sorte de synthèse biologique préfigurant l'écologie humaine, celles de A. Cholley, qui pressent à la fois la combinatoire naturelle et l'" analyse de système ", l'exemple de Ch. P. Péguy, qui propose une sorte de synthèse topologique du Queyras, restent à peu près sans suite. La géographie régionale, descriptive, non conceptualisée, n'est plus alors qu'un procédé littéraire sans avenir. De plus, la mise en situation brutale de faits humains dans un «cadre naturel "dessiné a priori fait peser une lourde ambiguïté sur l'ensemble de la démarche géographique. L'école française s'est laissée emporter, en dépit de réticences, et même de résistances, presque unanime, par une méthode d'analyse fondamentalement détermi-

1. G. Bertrand (2). Le numéro entre parenthèses renvoie à l'orientation bibliographique in fine. 
niste qu'elle ne dominait pas. Le milieu naturel étant alors traité dans une description à tiroirs, qui se développe en porte-à-faux par rapport à la problématique sociale, la géographie humaine se détourne de plus en plus de ce type d'analyse à la fois équivoque et inutile. Inapte à saisir le paysage dans sa complexité socio-écologique, la géographie classique n'en apportait pas moins quelques contributions indispensables à l'analyse paysagère : les notions d'espace et d'échelle, l'apport de la géomorphologie et la familiarité avec les sciences historique et archéologique.

\section{Le géosystème et la maîtrise du " sous-système naturel ".}

La recherche sur le paysage démarrant, en France, vers 1965 participe d'un mouvement de remise en cause et de renouvellement de la recherche scientifique. A cette convergence épistémologique conduisaient diverses "filières »: le développement de la Biologie, puis de l'Ecologie biocénotique, qui démontre l'unité biologique et fonctionnelle de la nature en imposant les premiers concepts intégrateurs (biocénose, biotope, écosystème); les modèles du structuralisme linguistique; la théorie des ensembles et l'analyse systémique qui donnent une réalité aux complexes et permettent de les examiner globalement sans les détruire; la réflexion sur la Nature retrouvée dans ses rapports avec la culture et la société par les sociologues et les anthropologues; l'effort plus général d'insertion de l'Ecologie dans l'analyse marxiste.

A l'origine des travaux modernes sur le paysage « naturel » sont les puissantes méthodologies mises au point par les géographes soviétiques et généralisées en Pologne, Tchécoslovaquie, Allemagne, où elles se combinent avec le courant de la Landschaftsökologie (2). Les méthodes utilisées sont essentiellement quantitatives et fondées sur la cybernétique. Elles s'appuient sur des mesures stationnelles qui permettent d'établir des bilans biogéochimiques et énergétiques à l'échelle des différents " géofaciès " du paysage. Il existe une "science du géosystème », selon Sochava, c'est-à-dire une "science du paysage naturel " qui, permet non seulement de dresser des bilans mais s'exerce déjà, grâce à la cybernétique, à la prévision des « états " du paysage. Les études de "landscape survey " réalisées depuis le début du $\mathrm{XX}^{*}$ siècle dans plusieurs pays anglo-saxons, à la fois plus « légères » et plus pragmatiques, débouchent directement sur l'aménagement de l'espace.

La méthode de recherche proposée à Toulouse dès 1967 et affinée depuis, s'inspire de ces différents courants. Elle repose sur quelques principes élémentaires :

2. N. Beroutchachvili et G. Bertrand. Le géosystème ou système territorial naturel. $R G P S O, 1978,2$, supra, p. 167 sqq. 
C'est une méthode globale. Le paysage dit « naturel " est à la fois une structure et un système qui fonctionne dans l'espace et dans le temps. Adéquation d'une méthode à son objet, mais aussi réaction contre la propension naturelle de la recherche scientifique à se fragmenter et à perdre de vue ce qui est essentiel pour la connaissance des combinaisons écologiques ou sociales.

C'est une méthode intégrée. L'intégration n'étant pas la synthèse et l'analyse du paysage n'étant pas une "super-synthèse " encyclopédique, il s'agit d'une analyse de système qui reconnaît une hiérarchie entre les ensembles et les éléments et en étudie les interrelations.

C'est la recherche d'un nouveau langage. Sans un tel langage l'objet-paysage ne saurait avoir d'existence pratique et scientifique, d'où la priorité accordée à la définition des unités de paysage et à leur classification. De simples typologies compréhensives, on est passé à des systèmes taxo-chorologiques (J.F. Richard) plus élaborés (3) qui permettent de saisir toutes les nuances de la mosaïque paysagère.

Les notions d'échelle des phénomènes naturels ou humains et de seuils dans l'espace et dans le temps sont, par définition, indissociables de la taxinomie et de la chorologie. Des recherches sur les « états » des géosystèmes permettent, d'une part, de définir chaque unité paysagère par son rythme (phénologie) et, d'autre part, de suivre et même de prévoir les évolutions du paysage. On dispose ainsi des prémices d'une histoire et d'une archéologie du paysage (4).

Le géosystème permet d'appréhender sans discrimination et sans hiérarchisation préalables la totalité du « complexe géographique naturel ». Le «sous-système naturel " qui fonctionne dans chaque paysage peut donc être parfaitement maîtrisé en lui-même et pour lui-même. Mais le problème méthodologique du paysage n'est pas pour autant résolu.

\section{Le paysage sur la marge de l'écologie.}

L'écologie domine les sciences de la nature et pénètre au plus profond des sciences humaines et sociales. Faut-il y voir un progrès méthodologique dans la mesure où des concepts éprouvés tels que l'écosystème, l'environnement, etc., paraissent aussi bien s'appliquer à la société qu'à la nature ? L'écologie scientifique a-t-elle réussi là où la géographie a échoué ? Y a-t-il une solution écologique à la problématique paysagère?

3. J.F. RICHARD (16).

4. G. BI:RTRAND. L'archéologie du paysage dans la perspective de l'écologie historique. Caesarodumum, n ${ }^{\circ} 13,1978, \mathrm{p} .132-138$. 
La recherche sur le paysage s'inscrit dans le courant général de l'écologie scientifique, mais sur la marge et dans une attitude de réflexion critique, les réserves ne portant pas sur la méthode écologique proprement dite, mais sur les modalités théoriques et pratiques de son application au domaine social. L'écologie est une science biocentrique et l'écosystème est un concept biologique qui focalise le bilan énergétique sur la production de matière vivante par le mécanisme de la photosynthèse, du métabolisme et de la chaîne alimentaire. L'environnement, sous-produit de l'écosystémique, est donc lui aussi, à l'origine, un concept biologique.

Sur le plan méthodologique, l'analyse écosystémique est souvent directement assimilée à une analyse de système et considérée comme un simple instrument de travail " neutre ", sans que l'on perçoive les interférences possibles avec un finalisme biologique. Par exemple, les processus et les éléments abiotiques sont subordonnés aux processus et aux éléments vivants; or, le végétal ou l'animal ne sont ni le centre du paysage ni même le "réactif »privilégié de ce dernier.

Sur le plan épistémologique, l'écologie peut représenter une intrusion du scientisme dans l'analyse sociale. Si l'écologie, plus qu'aucune autre discipline naturaliste, nourrit très directement la réflexion philosophique, elle ne peut prétendre pour autant remplacer la philosophie. Si l'écologie est en passe de maîtriser les aspects biologiques de l'environnement social, - voir les progrès récents de l'éthologie humaine et de l'écologie médicale -, elle n'en pêche pas moins par naïveté et réductionnisme chaque fois qu'elle prétend habiller la réalité sociale de ses propres concepts et imposer une interprétation globale - écosystémique - de la société humaine; en témoigne la tentative de synthèse écologique de P. Duvigneaud (5). Alors, le biologisme fonctionnaliste n'est pas très loin, ouvrant la porte au prophétisme et au catastrophisme écologiques. On comprend donc la réserve de principe adoptée par certains écologues et, plus systématiquement, par l'ensemble des chercheurs marxistes qui intègrent, lentement, l'écologie dans l'analyse des processus sociaux.

Les ambiguïtés de l'écologie se retrouvent dans les avatars du concept d'environnement élargi au domaine social qui a rapidement perdu sa cohérence physique pour devenir une notion aussi vaste que mal formulée, alpha et oméga d'une écologie sociale qui joue à nouveau avec un certain déterminisme naturel.

L'insertion du paysage dans l'analyse sociale ne passe pas, au moins directement, par des méthodes d'inspiration naturaliste. La société reste coupée de la nature, malgré les progrès considérables réalisés sur le plan philosophique et méthodologique et le

5. P. Duvigneaud. La synthèse écologique. Paris, Doin, 1974, 294 p. 
succès de quelques expériences pratiques, peu nombreuses et surtout trop sectorielles ou monographiques (6). L'alternative est encore entre la séparation ou la confusion des savoirs. Nous ne souhaitons proposer aucun passage direct entre l'analyse naturaliste et l'analyse sociale. Une méthode et un concept se dégradent quand ils transgressent leur propre champ disciplinaire, car ils changent implicitement de finalité et de contenu. Les concepts de géosystème et d'écosystème ne sont pas transposables à l'analyse sociale. Ils ne peuvent servir de point de départ à l'étude du paysage. Cependant, il faut y voir des analyses de systèmes réussies dans leurs domaines respectifs. Et ils peuvent servir d'exemples et de modèles analogiques. De plus, comme ils permettent de réduire la complexité du milieu naturel sans le mutiler, ils sont indispensables pour assurer l'insertion du naturel dans l'analyse sociale. Il ne peut $y$ avoir de projet pertinent que s'il est constamment étayé par une réflexion sur la nature et sur la place qu'on lui reconnaît par rapport à la culture et à la société. C'était le sens profond du vieux débat sur le déterminisme naturel; mais il était si mal posé qu'il a été progressivement éludé. L'une des plus graves lacunes de la pensée écologique contemporaine n'est-elle pas justement de débattre de questions sociales sans avoir assuré au préalable ses propres bases de références dans un domaine qui lui est étranger?

En reconnaissant a priori la réalité socio-écologique du paysage, on situe la réflexion sur l'interface nature-société. Comme l'écrit S. Moscovici, " le combat se déroule effectivement sur deux fronts. Sur le front des sciences sociales, pour leur faire connaître la dimension naturelle des phénomènes, des dynamismes sociaux. Sur le front des sciences naturelles, pour les amener à se pénétrer de la spécificité du social, à abandonner le mépris dans lequel elles tiennent l'acquis dans ce domaine et à renoncer aux simplifications, parfois puériles - elles abondent en éthologie - de leurs spéculations".

\section{La dialectique du paysage}

"Le principe de simplification a régné sur l'univers. Les choses ont été totalement et par principe isolées de leur environnement et de leur observateur, privés l'un et l'autre de toute existence, sinon perturbante. La concordance des observations élimina l'observateur et l'isolement expérimental élimina l'environnement perturbateur. Les choses devinrent objectives... ", comme l'établit E. Morin (7).

6. S. Moscovici (11).

7. E. Morin (10). 
Pris tantôt comme sujet, tantôt comme objet, souvent limité au phénomène de la perception, le paysage a échappé à la rationalité linéaire, réductrice et causaliste du cartésianisme ainsi qu'à l'objectivisme du scientisme positiviste. Dans l'émiettement de la recherche scientifique actuelle, l'analyse globale du paysage apparaît comme la poursuite d'une utopie dans un monde extradisciplinaire. Le but n'est pas d'aboutir, du moins dans l'immédiat, à une méthode d'analyse spécifique mais de soulever des problèmes que des démarches plus classiques et plus sectorielles ne permettent pas d'aborder. Toutefois, la logique du raisonnement implique que cette recherche, même superficielle et incertaine, soit conduite à son terme, c'est-à-dire qu'elle débouche sur une pratique de l'analyse paysagère. Le paradigme systémique ( $L$. von Bertalanffy, E. Morin, J.L. Le Moigne) (8) permet de tenter cette aventure. Il faut, dans un premier temps, organiser le paysage à l'intérieur d'un système. Appréhender un paysage, c'est accumuler consciemment les obstacles conceptuels et méthodologiques et s'attaquer à ce qui paraît être un tissu de contradictions. Il suffit d'énumérer les principales "qualités " que l'on reconnaît habituellement au paysage pour constater qu'elles relèvent de catégories souvent considérées comme étrangères ou contradictoires. Il s'agit donc de poser quelques postulats qu'on s'efforcera par la suite de démontrer :

1. Le paysage s'impose dans sa triviale globalité : il faut partir du banal et du global en se débarrassant (dans la mesure du possible) de tout présupposé disciplinaire, méthodologique et finaliste.

2. Le paysage est un objet socialisé, une image, qui n'existe qu'au travers du phénomène physiologique de la perception et d'une interprétation socio-psychologique. Forestiers et pasteurs vivent deux images différentes, sinon contradictoires, de la même forêt.

3. Le paysage n'en est pas moins une structure naturelle, concrète et " objective », c'est-à-dire indépendante de l'observateur. La forêt est un espace concret et une biomasse qui fonctionne sur elle-même.

4. L'image sociale du paysage est le produit d'une pratique économique et culturelle. Les forestiers posent sur la forêt le regard du maître des lieux devant un appareil qui produit du bois dans l'harmonie de la nature. Le regard des pasteurs, c'est celui de l'exclu devant un pâturage potentiel...

5. La spécificité du paysage vient moins d'être plus «complexe» et plus " hétérogène " que les objets scientifiques habituels, que de chevaucher les grandes catégories métaphysiques: le naturel et le culturel, l'espace et le social, l' " objectif » et le "subjectif ".

Avant d'envisager le système dans toute sa complexité, il faut s'interroger sur la signification épistémologique et sur les consé-

8. L. VON BERTALANFFy (1). 
quences méthodologiques de la coupure entre nature et société qui traverse le paysage. Par essence, le paysage relève d'une dialectique entre des lois physiques et des " lois » sociales.

\section{Le refus du manichéisme paysager.}

La plupart des études sur le paysage en sont restées, au moins implicitement, à une interprétation dualiste.

a. Le paysage est une nature-sujet. Il n'a d'existence sociale qu'au travers d'un processus passant de la formation de l'image à son interprétation sociale. Cette combinatoire qui fait intervenir différents mécanismes physiologiques, psychologiques, linguistiques, économiques, idéologiques, etc., a été maintes fois analysée et il ne paraît pas utile d'y revenir ici. Le paysage est défini comme un phénomène culturel.

b. Le paysage est une nature-objet. C'est une réalité qui existe indépendamment de l'observation et de l'observateur et qui n'est pas autre chose qu'une portion de l'espace terrestre. Le paysage est reconnu comme un phénomène naturel.

Ces deux propositions sont généralement considérées comme contradictoires: affrontement entre le culturalisme et le naturalisme, soit entre philosophie idéaliste et philosophie matérialiste. Dans ces conditions, la situation paraît effectivement sans issue.

Mais seule est sans issue l'interprétation idéaliste qui rétrécit le paysage à un simple phénomène de perception, limitant l'étude aux analyses $d$ ' " espaces perçus " ou d' " espaces vécus ". C'est d'ailleurs ce cheminement idéaliste, qui ne dit pas toujours son nom, peutêtre parce qu'il ne se reconnaît pas lui-même, qui l'emporte aujourd'hui dans la recherche sociologique et géographique (9). L'intérêt récent apporté au phénomène de la perception a permis de nuancer et d'humaniser - ou plus exactement de socialiser la prise en compte de l'environnement dit naturel. Progrès sur la géographie régionale classique, cette démarche n'en présente pas moins trois défauts rédhibitoires (10).

L'existence matérielle et "objective " du pavsage est niée, ou négligée. Dans la plupart des cas, il s'agit plus d'une réaction épidermique, auto-défense contre un usage mal compris du "déterminisme naturel "que d'une position de principe.

La société est isolée de ses fondements biologiques et spatiaux et le milieu physique, s'il est mentionné, demeure dans la confusion. Le système de production est coupé de sa base de production primaire. Economistes, sociologues et " new geographers " localisent

9. A. Frémont (5).

10. G. Brertrand. Pour une histoire écologique de la France rurale. Histoire de la France rurale. Paris, Le Seuil, 1975. 
le plus souvent leurs pôles et leurs flux d'activités sur des espaces géométriques et dénaturés.

Les notions d'espace vécu et d'espace perçu ne peuvent prétendre couvrir l'ensemble du phénomène culturel, ce " filtre de la civilisation ", selon P. Gourou, dont l'analyse réclame une projection historique que seuls ont su réaliser des ethnologues étudiant de petites communautés humaines qui exploitent directement un milieu bien circonscrit (11).

\section{L'interrelation objet-sujet.}

Aucun progrès ne sera réalisé sans la reconnaissance de la globalité du phénomène paysager et l'élaboration d'une méthode appropriée. La problématique se fonde sur quatre postulats.

1. Renoncer à la démarche idéaliste et à ce qui constitue, au moins dans la pratique actuelle, son principal corollaire, l'analyse sectorielle du paysage "vécu » ou « perçu ».

2. Considérer le paysage à la fois comme objet et sujet, réalité écologique et produit social.

3. Appréhender la relation objet-sujet dans un mouvement dialectique. "Sur le plan épistémologique général, remarque S. Moscovici, le sujet et l'objet ne sont plus des entités données qui se constituent chacune de son côté, mais des produits de l'expérience et de la théorie qui les définissent l'un par l'autre; ils se forment l'un par l'autre et sortent transformés de leur rencontre. De par leur structure, ce sont des mixtes. Leur différence et leur coupure n'a pas à être remise en question dans une unité molle et conciliante, puisqu'elle est la condition nécessaire à toute science. Cependant, il convient d'en donner un sens nouveau : le sens d'une affirmation simultanée du sujet et de l'objet - au lieu de la négation du sujet par l'objet et vice-versa - le sens d'une relation changeante des poids respectifs, suivant l'échelle et le caractère de la réalité à comprendre, la précision des vérités à obtenir » (12).

Une telle problématique du paysage englobe dans un même mouvement l'objet spatial et les différentes utilisations et perceptions liées aux différentes pratiques sociales.

4. Toutefois, la coupure entre analyse naturaliste et analyse sociale ne peut être évitée dans l'état actuel de la méthode scientifique; mais elle n'est ni permanente ni insurmontable. Des acquisitions récentes de l'anthropologie physique et sociale, ainsi que de l'éthologie animale et humaine, démontrent que non seulement cette distinction se déplace mais encore qu'elle s'atténue à mesure que la recherche progresse. Le paysage ne se trouve ni d'un côté ni

11. J. BARrac dans Eléments d'ethnologie de R. Cresswell. Paris, Colin, 1975,2, p. $7-41$.

12. S. Moscovici (11). 
de l'autre, ni entre les deux : il est dans les deux. Analyse naturaliste et analyse sociale représentent donc les deux "faces " explorées d'un même paysage.

\section{Recherche d'un paradigme socio-écologique}

La question est posée par J.-L. Le Moigne : Comment représenter par un système un objet identifiable par un observateur ?

Il existe plusieurs façons de concevoir l'étude du paysage. La problématique proposée vise à analyser le paysage à la fois dans la Nature et dans la Société, démarche impliquant la mise au point d'un nouveau paradigme, c'est-à-dire d'un ensemble de théories et de modèles explicatifs susceptibles d'appréhender globalement le paysage dans sa dimension socio-écologique.

\section{Le paysage est un système...}

Le plus simple et le plus banal des paysages est à la fois social et naturel, subjectif et objectif, spatial et temporel, production matérielle et culturelle, réel et symbolique, etc. Le dénombrement et l'analyse séparée des éléments constitutifs et des différentes caractéristiques spatiales, psychologiques, économiques, écologiques, etc., ne permettent pas de maîtriser l'ensemble. La complexité du paysage est à la fois morphologique (forme), constitutionnelle (structure) et fonctionnelle et il ne faut pas chercher à la réduire en la divisant. Le paysage est un système qui chevauche le naturel et le social.

Comment est organisé et comment fonctionne un système paysager ? Le paysage s'inscrit dans l'espace réel et correspond à une structure écologique bien déterminée; mais il n'est "saisi " et qualifié en tant que tel qu'à partir d'un mécanisme social d'identification et d'utilisation. Deux caractéristiques fondamentales du paysage sont ainsi mises en évidence.

Le paysage apparaît de moins en moins comme une structure écologique et sociale et de plus en plus comme un processus de transformation, donc comme un phénomène inscrit dans l'histoire.

Le paysage est une interprétation sociale de la nature. Et la méthode consiste, au moins dans un premier temps, à aller de la Société vers la Nature (13).

Le postulat qui fonde l'analyse paysagère ne peut donc être que social : "c'est le système de production au sens large, c'est-à-dire produisant des biens matériels et culturels, qui, à l'intérieur d'un groupe social défini et dans un espace donné, dessine le contenu

13. E. MORIN (10). 
matériel et culturel d'un paysage ». Par groupe social, entendons un ensemble d'individus organisés à l'intérieur d'un même système de production, liés entre eux par une mèn:e pratique de la Nature et qui produisent un ensemble cohérent de biens matériels et culturels. Le paysage n'a donc de réalité et de sens que pour un groupe social et il peut y avoir pour une même structure matérielle « objective » autant de processus paysagers qu'il y a de groupes sociaux. Le système de production agro-sylvo-pastoral a mis en place à partir du milieu montagnard pyrénéen un ensemble paysager dont le contenu matériel (organisation des terroirs, phénologie, fertilités, productions animales et végétales) et la signification culturelle ne se définissent que par rapport à la société montagnarde. L'estive, pâturage d'altitude, est un géosystème ou un géofaciès défini par une certaine combinaison topoclimatique-pédologique-floristique. Mais c'est aussi un espace fortement socialisé, dominé par toute une pratique et toute une symbolique pastorales. L'estive est socialement signifiée, organisée autour de pôles (cabanes, jasses, abreuvoirs), parcourue de flux saisonniers (bergers et troupeaux). La source est un point d'eau, mais aussi un point de repère et de rencontre, parfois une point de neutralisation ou bien de lutte dans un espace conflictuel. Le paysage agro-sylvo-pastoral est au-delà de ce qui se voit; il est ce qui se sait, se fait, doit se faire, ce qui se vit quotidiennement : telle limite foncière invisible mais qu'on ne doit pas transgresser matérialise tel rapport de force à l'intérieur du groupe social, tel tabou spatial, tel projet de culture ou tel espoir de récolte. Le voyeur, le touriste ou le chercheur scientifique peuvent-ils, de l'extérieur, saisir mieux que des formes riches mais muettes?

L'analyse d'un paysage ne se justifie d'abord que par rapport à la société qui l'a élaboré et qui le vit dans les cadres technologiques et culturels de son système de production. Le paysage est donc un produit social qu'il faut situer par rapport aux productions naturalistes que sont le géosystème et l'écosystème.

Le géosystème et l'écosystème sont des concepts naturalistes qui incorporent plus ou moins directement certains aspects sociaux (impact anthropique); le paysage est une analyse sociale qui incorpore du " naturel » finalisé.

Le géosystème et l'écosystème sont des concepts directement quantitatifs fondés sur des mesures et sur l'établissement de bilans énergétiques; le paysage est intrinsèquement lié à l'idée de qualité qui s'exprime à partir d'un système de valeur social (bien que certains de ses composants soient quantifiables).

Le géosystème et, dans une moindre mesure, l'écosystème, sont des concepts spatiaux (chorologiques) alors que le paysage exprime premièrement un schéma de fonctionnement. Le paysage est un processus, produit du temps et plus précisément de l'histoire sociale. 


\section{2. “ La complexité est à la base "(E. Morin).}

Le processus paysager, développé à partir d'un faisceau d'interactions d'une telle complexité, peut être considéré comme un "polysystème " regroupant des systèmes eux-mêmes complexes mais bien individualisés et fonctionnant de façon plus ou moins autonome (système naturel et système social, système de production économique et système de représentation culturelle, etc.). «Rien clans le paysage ne change en même temps, à la même vitesse ou dans la même direction " (M. Santos) (14). Toute étude paysagère pose donc a priori le problème de l'analyse des décalages dans l'espace et des déphasages dans le temps entre les principaux composants du processus. Cette situation dynamique favorise les manifestations d'hystérésis et d'inertie. Les paysages actuels des centres de ville donnent un bon exemple de ces distorsions entre une situation socio-démographique héritée, des évolutions économiques contradictoires et des réalisations architecturales agencées dans le contexte d'une revalorisation mystificatrice du domaine urbain.

a. Déphasage entre le naturel et le social. Le fonctionnement naturel s'inscrit au-delà de la longue durée des groupes sociaux concernés et même de la durée de l'histoire humaine. Diverses ressources naturelles sont considérées comme "non renouvelables" à l'échelle humaine, soit qu'elles correspondent à un héritage géologique (minerais, nappes d'eau souterraines, altérites, etc.), soit que leur mise en place exige des siècles (sols, forêts, etc.). Une altérite, un sol et une lande, combinés dans un même paysage, n'ont pas la même signification temporelle, en particulier du point de vue de leur utilisation économique. Ce déphasage banal n'est pas directement appréhendé par la "lecture " du paysage. Le chercheur doit passer par l'analyse géosystémique. Le paysan, par contre, disposant de l'expérience pratique du milieu, intègre directement et plus ou moins efficacement ce déphasage dans sa vision du paysage.

b. Déphasage entre l'élaboration des éléments matériels du système de production et l'élaboration des structures mentales. Les représentations sociales du paysage s'enracinent au plus profond dans la mémoire collective et l'imaginaire. La forêt de Brocéliande survit à son défrichement (15). Le paysage, patrimoine culturel et économique, est assumé comme un héritage. La maison familiale, la propriété ou l'exploitation agricole font partie du paysage. Per-

14. M. Sintos. De la société au paysage. Hérodote, no 9, 1978.

15. G. Birrtrand. Pour une histoire écologique... note 10, p. 247. 
manence culturelle qui favorise un certain respect du passé et qui entre souvent en opposition avec les impératifs du changement économique et social. Le paysage, transcendé par sa charge émotionnelle, relève le plus souvent de l'archéologie sociale.

c. Stratification sociale du processus paysager. La division du travail et la diversification culturelle dans une même société font naître et s'affronter des relations matérielles et des représentations culturelles différentes pour un même espace.

Chaque société sécrète une interrelation paysagère privilégiée imposée à l'ensemble social par l'idéologie de la classe dominante. Telle la campagne, récemment perçue comme un espace vert de loisir. Telle la "réhabilitation " psycho-sociale du centre des villes.

Chaque groupe social vit un paysage spécifique dont le contenu, l'extension et la polarisation dépendent essentiellement de l'organisation du travail et des niveaux culturels. Tel ce paysage " moyen ", né de l'association d'un paysage urbain de HLM collectif et d'un paysage de camping de bord de mer. Mais les paysages catégoriels sont filtrés par l'image du paysage du groupe social dominant et parfois brouillés par ses représentations: l'image des " marinas " n'est pas étrangère à l'entassement estival dans les campings littoraux.

d. Le paysage éclaté. Dans les sociétés techniquement développées, fortement diversifiées et très inégalitaires, la relation privilégiée entre une société et un espace n'est plus qu'un souvenir assorti parfois d'un regret vague mais tenace. Les groupes sociaux ne sont plus contenus dans un paysage spécifique, riche de leur propre histoire. Le paysage est éclaté en fragments d'espaces spécialisés, sans liens historiques, souvent géographiquement isolés les uns des autres : espace pour travailler, espace pour habiter, espace de loisir. Ces unités monospécifiques qui manquent de profondeur historique et sociale gagnent rapidement sur les paysages traditionnels, en fondant un nouveau type de rapport entre la société et l'espace et constituant une nouvelle source de conflits entre les groupes sociaux.

\section{Le paysage mis en scène}

Conservons au paysage sa globalité de processus socio-écologique en l'analysant dans son environnement social et naturel et dans une perspective historique. Il faut affronter cette complexité sans verser dans des faux-fuyants sectoriels. Proposons, à titre provisoire, une démarche simple, tout en reconnaissant qu'elle relève largement de l'artifice. 


\section{Le scénario paysager et la règle des trois unités.}

Comme tout processus, le paysage n'a pas d'existence en dehors du système dans lequel il fonctionne. Elaborer une " mise en scène " du paysage, consiste à l'enfermer dans un système de référence socio-écologique. Pour retrouver la logique interne du système, on s'inspire directement d'une règle des trois unités :

Unité d'action, (ou de production), fondée sur le fonctionnement du système de production matériel et culturel. (Exemple : le système agro-sylvo-pastoral pyrénéen).

Unité de temps correspondant à une période stable du système de production. (Exemple, le système agro-sylvo-pastoral pyrénéen entre le début du déclin démographique (1850-1870) et la mise en place des premiers grands équipements touristiques (1950-1960).

Unité de lieu, délimitant l'espace matériel où se développe le système de production. Elle peut, dans les cas les plus simples correspondre au paysage lui-même (Exemple : paysage du système agrosylvo-pastoral pyrénéen) ou bien s'en distinguer, s'il s'agit de paysages éclatés.

Le paysage est alors inséré dans un réseau cohérent de signifiants sociaux. Ce système de référence sociale incorpore l'utilisation matérielle et la signification culturelle du paysage, qui peuvent être de moins en moins dépendantes l'une de l'autre. Le paysage, devenu un produit social finalisé, ne cesse pour autant de constituer une réalité écologique. Son contenu socio-écologique et son enveloppe écospatiale peuvent être délimités. Dans cette perspective, la "vocation naturelle " et le " déterminisme naturel "s'effacent devant la détermination technique et culturelle. Le déterminisme naturel (16) devient alors un déterminisme social qui joue à plein dans la production du paysage. Que le système de production change, toute la relation paysagère en sera modifiée, hormis quelques éléments qui peuvent se maintenir par inertie et constituer autant de cas d'hystérésis. Des déterminismes s'effacent, d'autres apparaissent, aussi provisoires que les systèmes de production dont ils dépendent. Le paysage n'est plus cette entité neutre et compassée qui surplombe immuablement le champ social. C'est un produit économique et culturel intégré au système social, mais il ne cesse d'apparaître et de fonctionner comme un système écologique.

Toutefois, ce scénario ne s'applique en tant que tel que dans le cas de sociétés agraires fortement enracinées dans le temps et dans l'espace, telles les sociétés rurales d'Ancien régime en France ou les sociétés montagnardes au Népal avant l'explosion démographique de la première moitié du $X^{\prime \prime}$ siècle. Cela permet de définir un type de rapport pré-industriel et pré-urbain dans lequel le paysage se confond avec l'espace de production et avec l'espace quotidien-

16. G. Bertrand. Pour une histoire écologique.... 
nement vécu. Dans ces « agrosystèmes " presque immobiles et fermés sur eux-mêmes, le déterminisme socio-écologique joue pleinement et la marge d'innovation, très réduite, reste le privilège de la classe dominante qui peut seule prétendre à d'autres expériences à partir de moyens extérieurs au système socio-écologique ou bien prélevés sur ce dernier.

\section{Les scénarios complexes.}

L'élaboration du scénario doit être modulée en fonction de la complexité croissante des systèmes sociaux. D'où la nécessité d'un système de scénarios emboîtés et dépendants les uns des autres à la fois dans l'espace social et dans le temps.

a. Le scénario paysager dominant représente le modèle économique et culturel dominant. Ce modèle impose à toute une société un même type de relation avec le paysage et en favorise la diffusion et la reproduction. Dans la société caractérisée aujourd'hui par la concentration de la population dans des sous-paysages industriels et urbains de plus en plus contraignants, se développe, comme en antidote, une idéologie du "retour à la nature et à la vie naturelle » qui impose des modèles de consommation des paysages.

b. Les sous-scénarios, ou scénarios paysagers dominés, expriment la situation réelle des différentes catégories sociales dans leur pratique économique et culturelle de l'espace. Ces sous-scénarios peuvent être eux-mêmes plus ou moins emboîtés pour traduire les multiples nuances sociales. Tel, le cas des travailleurs immigrés vivant leur déracinement paysager dans d'infra-paysages urbains.

c. La relation entre le processus paysager dominant et les divers processus paysagers dominés ne peut s'expliquer que dans le temps. Elle impose l'élaboration de scénarios historiques qui permettent d'étudier, par grandes catégories sociales, les problèmes de décalage et d'hystérésis. L'analyse du système pavsager dans une société donnée revient à établir l'organigramme des scénarios paysagers en fonction des catégories sociales en présence. Ce que nous avons développé à propos du Sidobre.

Il ne paraît pas encore possible de réduire la multitude des scénarios paysagers à quelques catégories simples et d'en dresser la typologie. Toutefois, la « méthode »des scénarios a déjà fait perdre au paysage son caractère d'imprécision littéraire et de phénomène " exceptionnel ». Tous les scénarios sont construits sur des modèles identiques; ils sont donc comparables, soit globalement, soit terme à terme (scénario dominant, sous-scénarios hiérarchisés, etc.). Nous demeurons loin d'une généralisation taxochorologique comparable à celle du géosystème; mais, au-delà de l'évocation littéraire monographique, est amorcée une première description raisonnée, étape initiale de la méthode comparée. 


\section{En guise d'introduction à l'analyse du paysage}

La découverte du paysage par un nombre de plus en plus grand de disciplines, la résurgence du paysage dans des littératures géographiques fort différentes semblent indiquer que le terme n'est plus suspecté ici de conservatisme et là d'aventurisme. Le paysage rentre dans l'ordre scientifique après avoir été tenu en lisière. Ce qui devrait réjouir un ancien utilisateur de la terminologie paysagère (17) si, au-delà des pétitions de principe, pouvaient s'amorcer une réflexion conceptuelle et une recherche pratique.

\section{Eléments pour une définition du paysage.}

Le paysage est-il, très précisément et tout simplement, ce qui se voit et qui n'est justement ni précis, ni simple ? Le paysage n'est-il, comme pour Y. Lacoste, qu'une "vision des trois dimensions, qui d'un certain point d'observation ne peut pas tout voir, parce que certaines parties d'espace sont masquées »? Mais si la perception est, nécessairement, à l'origine de la représentation d'un paysage, elle ne suffit pas à expliquer cette dernière.

Proposer une définition, toujours mutilante, serait, de toute façon, prématuré. Mais avançons quelques éléments qui ne peuvent encore prétendre constituer à eux seuls, un système explicatif et encore moins aboutir à l'élaboration d'un concept. La confusion qui règne à propos du paysage vient pour une part de ce que l'on confond le mécanisme qui est à l'origine de la formation d'un paysage avec la signification du paysage lui-même et son interprétation sociale.

La production d'un paysage est généralement envisagée comme un processus tripolaire dans lequel interviennent un observateur, un mécanisme de perception, un objet.

A la base du paysage, une portion d'espace matériel existe en tant que structure et système écologique, donc indépendamment de la perception. Matérialité préalable qui enracine le paysage dans l'espace et détermine une enveloppe et un contenu communs à toutes les représentations paysagères de cette portion d'espace.

L'observateur individuel participe d'un système historico-culturel et socio-économique qui canalise ses interprétations paysagères. Il faut reconnaître une production sociale du paysage qui est diverse et contradictoire (scénarios), car toutes les catégories sociales n'ont pas le même accès culturel et matériel au paysage.

La perception directe d'un espace, qui permet la représentation du paysage doit être resituée dans un processus plus complexe de type dialectique qui lie l'observateur à l'espace et inversement.

17. G. Bertrand (2). 
Nous ne pouvons raisonner comme si c'était toujours la première fois qu'un observateur enregistre un paysage. Ce dernier, ou du moins le type de paysage correspondant, est immédiatement, sinon préalablement, décrypté, filtré, conditionné par la mémoire individuelle et collective. Le développement des moyens de diffusion audio-visuels a fortement accéléré ce processus. Le contact avec le terrain est sans doute indispensable au déclenchement du processus paysager, mais il n'est pas essentiel dans la production finale de l'image. A vouloir définir le paysage à partir d'une lecture directe d'un espace, on retomberait dans l'un des dogmes de la géographie traditionnelle. A l'idée de paysage saisi grâce à la vision instantanée du terrain, il faut substituer le lent cheminement d'un processus qui est historique et social bien avant d'être individuel et personnel. Ethnologues et anthropologues ont depuis longtemps analysé les reproductions de l'espace spécifiques à certaines communautés humaines.

La conception du paysage qui se dégage ainsi progressivement reste encore assez vague et incertaine. Simple « notion ", plutôt que concept. Toutefois, il paraît intéressant de le rapprocher à nouveau des concepts d'écosystème et de géosystème. Le concept d'écosystème est l'interprétation biologique de l'interface terrestre, le concept de géosystème en est l'interprétation géochimique. Le paysage n'est-il pas, tout banalement, une interprétation sociale de l'interface terrestre que la recherche scientifique ne saurait encore que partiellement appréhender ? Le rapprochement de la notion de paysage avec la notion d'environnement devient alors très significative. L'environnement consistc dans l'ensemble des éléments externes qui entourent la société et qui interagissent avec elle; le paysage est, au contraire, une production interne, née de la société et conférant une existence sociale à ce qui se trouve au contact de l'enveloppe externe de celle-ci, c'est-à-dire l'interface société-nature.

Dans une telle perspective, le paysage s'individualise à la fois par rapport à l'espace proprement dit et par rapport au mécanisme de la perception. Il apparaît de plus en plus comme un produit social historisé qui permet d'interpréter l'espace géographique dans les limites d'un système de production économique et culturel. Le paysage est alors posé comme un médiat entre la société et la nature, soit une interprétation sociale de la Nature et peutêtre, au-delà, une interprétation naturelle de la Société.

\section{Le paysage et les contradictions du système socio-écologique.}

Considérant le paysage comme un des processus du polysystème socio-écologique, on met en évidence quelques contradictions qui placent le paysage au cœur de la problématique écologique et sociale. 
Contradictions entre l'état écologique réel d'un paysage et son interprétation sociale (certains milieux montagnards agro-sylvopastoraux considérés et traités comme " milieux naturels »).

Contradictions entre le modèle dominant de production et de représentation du paysage et la réalité vécue par les catégories ou classes sociales dominées (l'élitisme des aménagements d'espaces de loisirs).

Contradictions entre les besoins économiques et les modèles culturels imposés et, d'autre part, les possibilités matérielles et les aspirations des différentes classes sociales.

Au-delà des apparences et des images imposées, les paysages que nous produisons et qui nous entourent nous échappent encore dans leur profondeur historique, écologique, sociale. L'accès au paysage et à sa représentation culturelle ne constituent-ils pas un aspect des luttes sociales?

Le processus paysager n'est pas encore maîtrisé. En choisissant de situer l'analyse du paysage entre la Nature et la Société nous explorons, parmi d'autres, une voie encore peu fréquentée, mais qui offre la possibilité de resituer la Nature dans la dynamique sociale et la Société dans la dynamique naturelle. Au-delà de l'argument paysager nous retrouvons la complexité vécue du quotidien et du banal. 


\section{ORIENTATION BIBLIOGRAPHIQUE}

1. L. Von Bertalanffy, Théorie générale des systèmes, Paris, 1973, Dunod.

2. G. Bertrand. Paysage et géographie physique globale. RGPSO, 1968.

3. G. BERTRAND, La Géographie physique contre-nature? Géodoc 8. Institut de Géographie de Toulouse, 1978, 33 p. et Itinéraires en Géographie physique. Hérodote, à paraître.

4. R. Dubos, L'homme et l'adaptation au milieu, Paris, 1973, Payot.

5. A. Frémont, La région, espace vécu. Paris, 1976, PUF.

6. P. Gourou, Pour une géographie humaine. Paris, 1973, Flammarion.

7. Y. LAcoste, A quoi sert le paysage ? Hérodotc, 7, 1977.

8. F. JACOB. La logique du vivant. Paris, 1970, Gallimard.

9. J.-L. LE MoIGNE, La théorie du système général. Théorie de la modélisation. Paris, 1977, PUF.

10. E. Morin, La Méthode. I. La Nature de la Nature. Paris. 1977, Seuil.

11. S. MoscovicI. Essai sur l'histoire humaine de la Nature. Paris, 1968, Flammarion.

12. S. MoscovicI. Hommes domestiques et hommes sauvages. Paris, 1974, UGE $(10 / 18)$.

13. P.E. Opum, Fundamentals of ecology. Philadelphia/London/Toronto, 1971.

14. P.E. Odum, Ecologie. Paris, 1976, Doin.

15. J. Piaget. Le structuralisme. Paris, 1968, PUF.

16. J.F. Richard. Problèmes de géographie du paysage. Abidjan, 1972, ORSTOM.

17. J. de Rosnay, Le Macroscope. Vers une vision globale. Paris. 1975, Seuil.

18. G. Rougerie (G.), Les cadres de vie. Paris, 1975, PUF.

RÉSUMÉ. - Le paysage, qui occupe une place essentielle dans la vie quotidienne, ne relève d'aucune catégorie scientifique et n'est réductible à aucun concept. On a choisi d'étudier le paysage à la charnière des sciences de la nature et des sciences de la société. Il est défini comme une interprétation sociale, à partir d'un système de production économique et culturel, d'un objet matériel qui existe indépendamment de l'observateur. Le paysage doit donc être appréhendé en même temps comme sujet et comme objet, ou plus exactement comme le passage incessant de l'un à l'autre. Cette analyse complexe et dynamique du " processus " paysager permet d'esquisser les scénarios paysagers qui, d'une part, permettent de mieux cerner le paysage comme " système " socio-écologique et, d'autre part, de mieux l'insérer dans une démarche sociale.

SumMary. - LANDSCAPE BETWEen NATURE AND SOCIETY. Though it plays an essential part in our daily life, the landscape does not belong to any scientific category and it cannot be reduced to any single concept. The author proposes to study the landscape as a conception that is related to both nature and society sciences. Then landscape is defined as a social interpretation of a real fact which does exist without any consideration of the observer's will; such a social interpretation cannot be separated from the economic and cultural production system to which the observer belongs. Thence any landscape should be examined at the same time as a subject and as an object as well, or, more accurately, as a permanent interchange between these two categories. Only such dynamic complex analysis of the landscaping process enables the scientist to draw the landscapes' sketches that can be used to define the landscape as a socio-ecological system and to recognize its function on the social process as a whole. 